INPLASY

PROTOCOL

To cite: Song et al. Protocol for a systematic review and metaanalysis of Kang-ai injection for patients with Oesophageal cancer. Inplasy protocol 202080019. doi:

10.37766/inplasy2020.8.0019

Received: 06 August 2020

Published: 06 August 2020

Corresponding author: Jinyan Wang

wjylcxh@163.com

Author Affiliation:

Liaocheng People's Hospital

Support: 2016ZRA15065

Review Stage at time of this submission: The review has not yet started.

Conflicts of interest:

The authors declare that they have no competing interests.

\section{Protocol for a systematic review and meta-analysis of Kang-ai injection for patients with Oesophageal cancer}

Song, QP1; Yang, W2; Meng, Z3; Wang, JY4.

Review question / Objective: Is Kang-ai injection efficacy and safety for the treatment of patients with oesophageal cancer (OC)?

Condition being studied: Kang-ai injection, efficacy, safety, and oesophageal cancer.

Information sources: Electronic databases including relevant RCTs and high-quality prospective cohort studies were searched from PubMed, Web of Science (WOS), Medline, Cochrane Library, Google Scholar, Excerpt Medica Database (Embase), Chinese Biomedical Literature Database (CBM), China National Knowledge Infrastructure (CNKI), China Scientific Journal Database (CSJD) and Wanfang Database will be systematically searched for eligible studies from their inception to August 2020. Language is limited with English and Chinese.

INPLASY registration number: This protocol was registered with the International Platform of Registered Systematic Review and Meta-Analysis Protocols (INPLASY) on 06 August 2020 and was last updated on 06 August 2020 (registration number INPLASY202080019).

\section{INTRODUCTION}

Review question / Objective: Is Kang-ai injection efficacy and safety for the treatment of patients with oesophageal cancer $(\mathrm{OC})$ ?
Rationale: Kang-ai injection, a well-known plant-derived traditional Chinese medicine, has been widely applied as a promising adjunctive drug for oesophageal cancer (OC). However, the exact effect of Kang-ai injection remains controversial. Therefore, in this study, we aimed to summarize the 
efficacy and safety of Kang-ai injection for patients with advanced OC through the meta-analysis, in order to provide scientific reference for the design of future clinical trials.

Condition being studied: Kang-ai injection, efficacy, safety, and oesophageal cancer.

\section{METHODS}

Search strategy: To perform a comprehensive and focused search, experienced systematic review researchers will be invited to develop a search strategy. The plan searched terms are as follows: "Oesophageal cancer" or "esophageal cancer" or "esophagus cancer" or "shiguanai" or "shiguanzhongliu" or "OC" or "EC" and "Kang-ai injection" or "Kangai injection" or "Kang'ai injection" or "Kang'ai zhusheye" or "KA injection" or "KAl" et al. An example of search strategy for PubMed database shown in Table 1 will be modified and used for the other databases.

Participant or population: Patients must be cytologically or pathologically confirmed as having $O C$ at a clinically advanced stage. There will be no restrictions regarding age, gender, racial, region, education and economic status. Patients with other malignancies or non-primary $O C$ are not included.

Intervention: In the experimental group, advanced $O C$ patients must be treated with conventional treatment (including radiotherapy, chemotherapy, and targeted therapy) combined with Kang-ai injection.

Comparator: In the control group, OC patient treated with the same conventional treatment as intervention group.

Study designs to be included: All available comparative clinical trials that assessed the efficacy of Kang-ai injection in the treatment of OC patients will be included.

Eligibility criteria: This study will include randomized controlled trials (RCTs) or quasi-RCTs, and high-quality prospective cohort studies that investigated the efficacy and safety of Kang-ai injectionmediated therapy in patients diagnosed with advanced OC will be included in this systematic review. Articles without sufficient available data, non-comparative studies, non-peer reviewed articles, literature reviews, meta-analysis, commentaries, case reports and series, meeting abstracts, letter to the editor, editorials, and other unrelated studies will be all excluded from analysis.

Information sources: Electronic databases including relevant RCTs and high-quality prospective cohort studies were searched from PubMed, Web of Science (WOS), Medline, Cochrane Library, Google Scholar, Excerpt Medica Database (Embase), Chinese Biomedical Literature Database (CBM), China National Knowledge Infrastructure (CNKI), China Scientific Journal Database (CSJD) and Wanfang Database will be systematically searched for eligible studies from their inception to August 2020. Language is limited with English and Chinese.

Main outcome(s): The primary outcomes will include: I) Overall response rate (ORR, complete response + partial response) and disease control rate (DCR, complete response + partial response + stable disease); II) Overall survival (OS, which is defined as the time from the date of randomization to death from any cause); III) Disease-free survival (DFS, which is the time from date of random assignment to date of recurrence or death).

Additional outcome(s): Secondary outcomes will include: I) Quality of life (QoL) as evaluated by Karnofsky score; II) Immune function indicators: CD3+, CD4+, CD8+, NK cells percentage, and CD4+/ CD8+ cell ratios, and serum cytokines level (IL-2, IL-4, IFN-ץ and TNF-a); III) Treatmentrelated adverse effects: toxicity was graded from 0 to IV in severity on the basis of the World Health Organization (WHO) recommendations.

Data management: Two reviewers (Qingping Song and Wei Yang) will be responsible for the data extraction 
independently according to the Cochrane Handbook for Systematic Reviews of Intervention. The following data will be extracted from eligible literatures: I) Study characteristics and methodology: country of study, the first author's name, year of publication, randomization, periods of data collection, and follow-up duration, et al. II) Participant characteristics: sample size, tumor stage (staging of the tumor according to the AJCC TNM classification for $\mathrm{OC})$, age, gender, tumor size, inclusion and exclusion criteria, et al. III) Interventions: therapeutic means, dosage of Kang-ai injection, administration route and cycles, and duration of treatment, et al. IV) Outcome and other data: ORR, DCR, OS, DFS, QoL, immune indexes [(CD3+, CD4+, CD8+, NK cells percentage, and CD4+/CD8+ cell ratios, and serum cytokines level (IL-2, IL-4, IFN- $\gamma$ and TNFa)], and adverse effects, et al. *Dealing with missing data: we will attempt to contact the authors to request the missing or incomplete data. If those relevant data are not acquired, they will be excluded from the analysis.

Quality assessment / Risk of bias analysis: Two review authors (Qingping Song and Wei Yang) will independently assess the methodological quality of the included RCTs by using the following criteria described in the Cochrane Handbook for Systematic Reviews of Interventions: random sequence generation, allocation concealment, blinding of participants and personnel, blinding of outcome assessment, incomplete outcome data, selective reporting and other bias. Evidence quality will be classified as low risk, high risk, or unclear risk of bias. Effective Practice and Organisation of Care (EPOC) guidelines will be used to assess the risks of non-RCTs. Any disagreements will be resolved via discussion with a third researcher (Zhen Meng).

Strategy of data synthesis: The statistical analysis for the extracted data from the included studies was conducted by Review Manager 5.3 (Nordic Cochran Centre, Copenhagen, Denmark) and Stata 14.0 (Stata Corp., College Station, TX, USA) software. Cochrane's Q-test and 12-test were used to evaluate the statistical heterogeneity for the pooled results. $\mathrm{P}<0.1$ for the Chi2 statistic or an I2>50\% will be considered as showing considerable heterogeneity, and the random effects model was considered according to the DerSimonian and Laird method. Otherwise, a fixed effect model will be used to calculate the outcomes. Continuous data will be presented as standardized mean difference (SMD) with their confidence intervals (Cls). Dichotomous data will be recorded as risk ratio (RR) with $95 \%$ Cls. For survival outcomes, Hazard ratios (HRs) with corresponding $95 \%$ Cls will be extracted from trials or be estimated from Kaplan-Meier survival curves by established methods. A two-tailed $P<0.05$ was considered statistically significant.

Subgroup analysis: If the data are available and sufficient, subgroup and metaregression analysis will be conducted to explore the source of heterogeneity with respect to age, gender, tumor stage, region, therapeutic regimens and courses.

Sensibility analysis: Sensitivity analysis will be conducted to assess the reliability and robustness of the aggregation results via eliminating trials with high bias risk. A summary table will report the results of the sensitivity analyses.

Language: Language is limited with English and Chinese.

\section{Country(ies) involved: China.}

Other relevant information: I) Publication bias analysis: Funnel plot, Begg's and Egger regression test will be performed to analyze the existence of publication bias if 10 or more studies are included in this meta-analysis. If reporting bias is suspected, we will consult the study author to get more information. If publication bias existed, a trim-and-fill method should be applied to coordinate the estimates from unpublished studies, and the adjusted results were compared with the original pooled RR. II) Evidence evaluation: The evidence grade will be determined by using 
the guidelines of the Grading of Recommendations, Assessment, Development, and Evaluation (GRADE). The quality of all evidence will be assessed at four levels: high, moderate, low, and very low.

Keywords: Kang-ai injection; oesophageal cancer; meta-analysis; efficacy; safety.

Dissemination plans: We will disseminate the results of this systematic review by publishing the manuscript in a peerreviewed journal or presenting the findings at a relevant conference.

Contributions of each author:

Author 1 - Qingping Song Conceptualization, Data curation, Formal analysis, Investigation, Methodology, Resources, Software, Supervision, Visualization, Writing-original draft.

Author 2 - Wei Yang - Data curation, Formal analysis, Investigation, Methodology, Visualization, Writing-original draft.

Author 3 - Zhen Meng - Funding acquisition, Investigation, Methodology, Validation, Writing-original draft, Writingreview \& editing.

Author 4 - Jinyan Wang Conceptualization, Project administration, Resources, Software, Supervision, Validation, Writing-review \& editing. 